\title{
Effect of Honey on Orthodontic Tooth Movement and Osteoclastic Activity in Psychologically Stressed Animals
} ${ }^{1}$ Reem Abid, ${ }^{2}$ Muhammad Sharjeel Ilyas, ${ }^{3}$ Ghazala Rubi, ${ }^{2}$ Amna Ahsan, ${ }^{2}$ Sadia Shakeel, ${ }^{1}$ Arfa Ahmed

${ }^{1}$ Department of Orthodontics, de'Montmorency College of Dentistry, Lahore

${ }^{2}$ Department of Oral Biology, Post Graduate Medical Institute, Lahore

${ }^{3}$ Central Research Laboratory, Postgraduate Medical Institute, Lahore

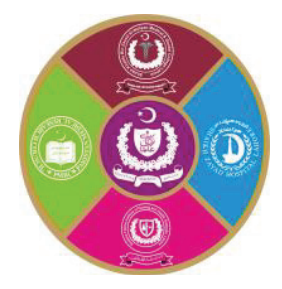

\section{ABSTRACT}

Introduction: Orthodontics is a clinical specialty in dentistry related to the correction of dentofacial deformities. Psychological stress factors delay Orthodontic tooth movement (OTM). Honey can be considered a useful and harmless natural product to reduce stress levels, hence improves the efficacy of OTM.

Aims \& Objectives: To compare the differences in the rate of tooth movement and osteoclastic activity between control, psychologically stressed and honey treated psychologically stressed groups after 1 week of orthodontic force application in an animal model.

Place and duration of study: This experimental study was conducted at the animal research laboratory and Histopathology Department of Post Graduate Medical Institute (PGMI), Lahore, Pakistan, from April 2019 to June 2020. Material \& Methods: Thirty-six Sprague Dawley rats were randomly divided into A, B and C groups. Psychological Stress was induced in group A (PS group) while Honey was given orally as a therapeutic agent along with induction of psychological stress in group B (PSH group), and group C was the Control Group. Statistical analyses were performed using SPSS version 24 software. The quantitative variables were the orthodontic tooth movement, the osteoclast count, and the expression of RANKL. One-way ANOVA was applied to calculate the mean difference and Post hoc Tukey test applied for multiple comparisons among the groups. A p-value $\leq 0.05$ was considered statistically significant in all 3 groups.

Results: There was a significant difference $(\mathrm{p}$-value $<0.05$ ) between control and experimental groups in the orthodontic tooth movement and levels of RANKL, however, there was no significant difference between PS and PSH groups.

Conclusion: Psychological stress delays orthodontic tooth movement by causing a reduction in its rate and osteoclastic activity and honey has no significant correlation with lowering stress levels, hence does not improve orthodontic tooth movement efficiency.

Key words: Orthodontic Tooth Movement (OTM), Psychological Stress, Honey.

\section{INTRODUCTION}

$\mathrm{M}$ alaligned teeth and jaw produce dentofacial deformity, which creates aesthetic problems, the continuous erosion of teeth, chewing, speech and sleep problems, and also causes psychological stress to the patients. ${ }^{1}$ Orthodontic tooth movement (OTM) helps ensure the proper function of teeth and solve aesthetic problems. It is a periodontally elicited biological response to mechanically applied forces ${ }^{2}$ Conventional orthodontic treatment demands a comprehensive duration of 18 to 30 months to get desired treatment outcomes. In this conventional method, the dynamic process of bone resorption and formation allows the tooth to move but it only depends on the interplay of the bone remodeling activities and the rates at which these activities take place. $^{3}$ The shortening of treatment duration with the introduction of biological as well as genetic therapies and removal of factors responsible for slowing the tooth movement is the main objective of the current advancements in the field of orthodontics. $^{4}$

Psychological or emotional stress is proven to be a risk factor in the pathogenesis and etiology of many diseases. ${ }^{5}$ Studies conducted to find out the role of psychological or emotional stress in the action, differentiation, and proliferation of osteoblasts, osteoclasts, and proinflammatory mediators during tooth movement are very limited though but conclusive of the fact that the psychological stress does affect orthodontic tooth movement at some point in time as it has effects on other body mechanisms. ${ }^{6}$ But to date, no study has been carried out to explore a stress reliever that can be harmlessly used by orthodontic patients routinely. Honey is the nectar of flowers processed naturally in the honeybees (Apis mellifera). ${ }^{7}$ Honey is beneficial due to its antibacterial, anti-inflammatory, antioxidant, hygroscopic, analgesic, and anxiolytic properties. Honey when applied externally can treat 
athlete's foot, eczema lip sores, and infected as well as sterile wounds caused by surgery, burns or accidents. $^{8}$ It can be used for the treatment of periodontal disease by its feature of being antiinflammatory. Honey contains antioxidant flavonoids (chrysin and gallic acid) that are capable of reducing stress and anxiety. ${ }^{9}$ Stress is undesirable in orthodontics as it demonstrates adverse effects on tooth movement. ${ }^{10}$

Cells of bone (osteoblasts) play an important role to regulate osteoclasts' differentiation for bone remodeling in OTM. Osteoblasts have firm control over osteoclasts' formation from monocytemacrophage lineage and trigger them to differentiate into osteoclasts in the presence of MCSF (c-Fms) and RANKL. ${ }^{9}$ The tooth displacement and remodeling of the bone is orchestrated by genetic as well as environment factors. ${ }^{11}$ The current research is a starter to broader studies in this field.

\section{MATERIAL AND METHODS}

Experimental Design: This experimental study was conducted at the Experimental Research Laboratory and Histopathology Department of Post Graduate Medical Institute (PGMI), Lahore, Pakistan, between April 2019 to June 2020. It was approved by the Institutional Review Board of (PGMI/LGH), Lahore, Pakistan vides. IRB\#: AMC/PGMI/LGH/ Article/ResearchNo00/52/21/ Date/ 25/06/2021.

A total of 36 Sprague Dawley rats ${ }^{12}$ of the female gender, weighing $(200 \pm 20 \mathrm{gms})$, age 10-12 weeks were obtained from Animal House PGMI, Lahore. The sample size was calculated by the following formula keeping the power of study equal to $80 \%$ and level of significance equal to $5 \% .^{12}$

All the work was carried out according to the National Institutes of Health guidelines. ${ }^{13}$ Male and diseased rats were excluded from the study. Female rats were selected because the hypothalamicpituitary-adrenal (HPA) axis and sympathoadrenal system of female rats are more reactive to stressful stimuli compared to male rats. ${ }^{14}$

Animals were divided randomly into three groups (Table-1) and each group was placed in separate cages which were labeled by tags. After the acclimatization of a period of one week, the experimental procedure was started.

Application of Stress Stimuli: The Predator Stress Effect was produced in animals of the PS and PSH group, as they were exposed to cat smell (incorporated in cloth by rubbing it in a wet state against the cat's body for few minutes), placed in the corner of each cage of both experimental groups for 20 days. The confinement stress stimulus was also applied by keeping animals of experimental groups in a crowded area having 12 rats in a cage of dimensions $(30 \times 20 \times 15 \mathrm{~cm})$ so that they were deprived of space for unrestricted movement area and were inescapably immobile. The Control group rats were kept in a less crowded environment ( 3 rats per cage of the same width). To remove bias, the control group cages were exposed to a clean but similar cloth for the same number of days. ${ }^{6}$

Application of Orthodontic Appliance: All animals were sedated with an intramuscular injection of Ketamine-xylazine $(10 \mathrm{mg} / \mathrm{Kg})$ on day 21 . The initial distance (T1) between the mesial occlusal pits of the right maxillary left first molar and the right maxillary second molar was calculated with the help of a digital Vernier caliper in all groups. A thin lace back wire $(0.9 \mathrm{~mm})$ was passed between two incisors to secure and hold the close coil spring around the teeth. An orthodontic close coil spring appliance between right maxillary $1^{\text {st }}$ molar and central incisors, having superelastic (Ortho Organizer., US) made up of nickel-titanium having a diameter $(0.010$ x $0.030 \mathrm{~mm}$ ), length $5 \mathrm{~mm}$, delivering consistent $20 \mathrm{cN}$ force (Fig-1) was secured in each animal. It was maintained for 7 days to produce orthodontic tooth movement in a molar tooth (Fig-1).

\begin{tabular}{|c|c|l|}
\hline Groups & $\begin{array}{l}\text { Number of } \\
\text { animals (n) }\end{array}$ & Assigned names. \\
\hline PS & 12 & $\begin{array}{l}\text { Experimental: Psychological stress } \\
\text { group (PS). }\end{array}$ \\
\hline PSH & 12 & $\begin{array}{l}\text { Experimental: Psychological stress group } \\
\text { with the honey application (PSH) }\end{array}$ \\
\hline Control & 12 & Control \\
\hline
\end{tabular}

Table-1: Details of animal groups

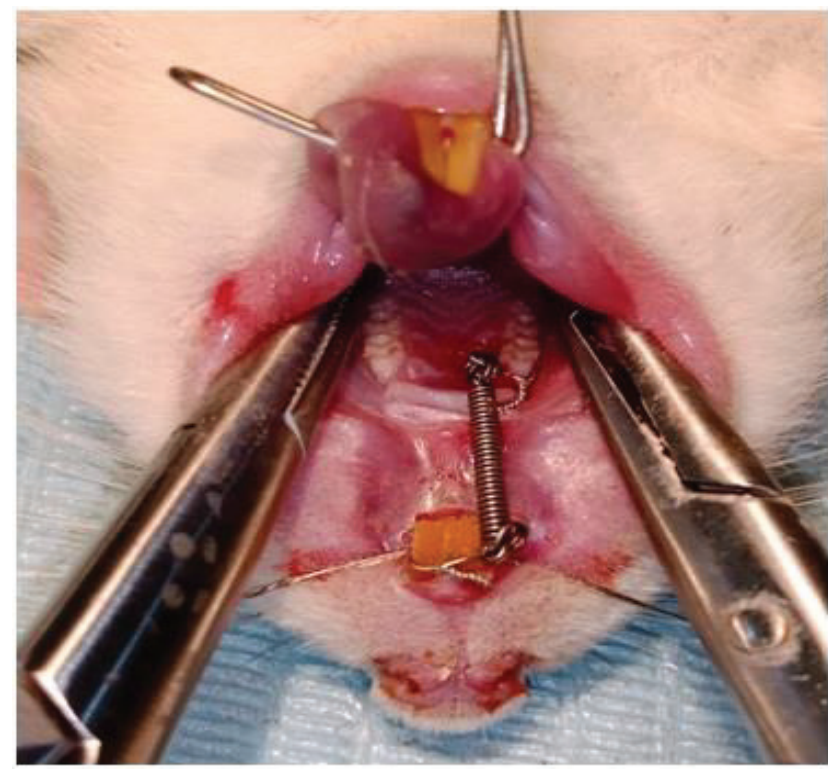

Fig-1: Appliance Secured between the first molars and incisors 
Application of Honey: In the PSH group, honey was administered orally daily $(0.5 \mathrm{ml} / 100 \mathrm{gm}$ b.w.). ${ }^{10}$ Honey preparation was done as $2 \mathrm{ml}$ of bee honey dissolved in $3 \mathrm{ml}$ of distilled water. This solution was given to the rats slowly paroral and it was made sure that the animals ingested the honey solution fully without dropping and wastage.

Analytical assays: The osteoclastic activity as a measure of orthodontic tooth movement was gauged through ELISA that quantifies the osteoclastic biomarker RANKL. Two $\mathrm{ml}$ blood was drawn on day 28 before sacrifice from the submandibular region on the working side in all three groups. Blood samples were collected in tubes containing EDTA, and serum samples were clotted at room temperature for $20 \mathrm{~min}$ and then centrifuged for $20 \mathrm{~min}$ at 2000-3000 RPM. Samples were allocated and stored at $-20{ }^{\circ} \mathrm{C}$ until analysis.

On day 28 , all the rats were euthanized by an overdose of chloroform. The maxillectomy of each rat was performed followed by complete separation of the palate. Maxilla having left the first molar was dissected and stored in $10 \%$ formalin solution. The final distance (T2) between the mesial occlusal pits of maxillary left first and second molars were measured on the dissected maxilla. The dissected maxilla was processed and stained with hematoxylin and eosin stain to quantify the number of osteoclasts, the mesial pressure side of a mesial root of maxillary left first molar where the appliance was placed was identified. Three sections for the histomorphometric study were selected by a histopathologist. These sections were quantified under 400x magnification in three consecutive microscopic fields.

\section{Statistical analysis:}

Statistical analyses were performed using The Statistical Product and Service Solution (SPSS) 24 software (IBM Corp., Armonk, NY, USA). The normality of the data was tested with Shapiro-Wilk tests. A one-way ANOVA test was applied to compare the initial distance, final distance, and orthodontic tooth movement, the expression of RANKL, and Osteoclastic cell count among groups. Post hoc Tukey analysis was performed for pairwise analysis in groups. The significance level was set at a $p$-value $<0.05$.

\section{RESULTS}

Orthodontic Tooth Movement (mm): The orthodontic tooth movement of rats in all groups was measured. A one-way ANOVA test was applied to compare the initial distance, final distance, and orthodontic tooth movement among groups. It was found that there was no significant difference in the mean initial and final distance among groups. However, the mean orthodontic tooth movement was significantly different among groups ( $\mathrm{p}$-value $<$ 0.001) (Table-2). For multiple comparisons, a post hoc Tukey test was applied, which showed that mean orthodontic tooth movement in the control group was significantly higher as compared to PS and PSH groups. However, no significant difference was found in orthodontic tooth movement between PS and PSH groups (Table-3).

\begin{tabular}{|c|c|c|c|c|}
\hline Variables & $\begin{array}{c}\text { Control } \\
\text { Group }\end{array}$ & $\begin{array}{c}\text { PS } \\
\text { Group }\end{array}$ & $\begin{array}{c}\text { PSH } \\
\text { Group }\end{array}$ & $\begin{array}{c}\text { p- } \\
\text { value\# }\end{array}$ \\
\hline $\begin{array}{c}\text { Initial distance } \\
\text { (T1) }\end{array}$ & $\begin{array}{c}2.87 \pm \\
0.40\end{array}$ & $\begin{array}{c}2.80 \pm \\
0.32\end{array}$ & $\begin{array}{c}2.88 \pm \\
0.22\end{array}$ & 0.781 \\
\hline $\begin{array}{c}\text { Final distance } \\
\text { (T2) }\end{array}$ & $\begin{array}{c}2.94 \pm \\
0.40\end{array}$ & $\begin{array}{c}2.83 \pm \\
0.33\end{array}$ & $\begin{array}{c}2.93 \pm \\
0.22\end{array}$ & 0.676 \\
\hline Distance & $0.68 \pm$ & $0.37 \pm$ & $0.48 \pm$ & $<$ \\
$(\mathrm{T}=\mathrm{T} 2-\mathrm{T} 1)$ & 0.14 & 0.12 & 0.17 & $0.001^{*}$ \\
\hline
\end{tabular}

$*_{p}$-value $\leq 0.05$ is considered statistically significant

Table-2: Comparison of orthodontic tooth movement among groups

\begin{tabular}{|c|c|c|c|c|}
\hline Group & Group & $\begin{array}{c}\text { Mean } \\
\text { Difference }\end{array}$ & $\begin{array}{c}\text { Std. } \\
\text { Error }\end{array}$ & p-value \\
\hline \multirow{2}{*}{ Control } & PS & 0.308 & 0.0597 & $<0.001^{*}$ \\
\cline { 2 - 5 } & PSH & 0.192 & 0.0597 & $0.008^{*}$ \\
\hline PS & PSH & -0.117 & 0.05971 & 0.140 \\
\hline
\end{tabular}

$* p$ value $\leq 0.05$ is considered statistically significant.

Table-3: Pair wise comparison of orthodontic tooth movement among groups

Osteoclastic Count in field of $5 \mathbf{m m}^{2}$ : The osteoclastic count in field $5 \mathrm{~mm}^{2}$ of rats in all groups was measured. It was found that there was no significant difference in the mean osteoclastic count among groups ( $\mathrm{p}$-value $=0.128$ )

Mean Levels of RANK-L (pg/ml): The mean levels of RANK-L $(\mathrm{pg} / \mathrm{ml})$ of rats in all groups were measured. A one-way ANOVA test was applied to compare the mean levels of RANK-L among groups. It was found that there was a significant difference in the mean levels of RANK-L ( $\mathrm{pg} / \mathrm{ml})$ among groups $(p$-value $=0.008)($ Table-4). For multiple comparisons, a post hoc Tukey test was applied which showed that mean levels of RANK-L $(\mathrm{pg} / \mathrm{ml})$ in the control group were significantly higher as compared to PS and PSH groups. However, there was no significant difference between PS and PSH groups (Table-4). 


\begin{tabular}{|c|c|c|c|c|}
\hline \multicolumn{5}{|c|}{ Comparison of mean levels of RANK-L (pg/ml) } \\
among groups
\end{tabular}

Pair wise comparison of mean levels of Rank-L (pg/ml) among groups

\begin{tabular}{|c|c|c|c|c|}
\hline \multirow{2}{*}{ Group } & Group & $\begin{array}{c}\text { Mean } \\
\text { Difference }\end{array}$ & $\begin{array}{c}\text { Std. } \\
\text { Error }\end{array}$ & $\begin{array}{c}\text { p- } \\
\text { value }\end{array}$ \\
\hline & PS & 0.308 & 0.0597 & $\begin{array}{c}< \\
0.001^{*}\end{array}$ \\
\cline { 2 - 5 } & PSH & 0.192 & 0.0597 & $0.008^{*}$ \\
\hline PS & PSH & -0.117 & 0.05971 & 0.140 \\
\hline
\end{tabular}

$*$ p-value $\leq 0.05$ is considered statistically significant

Table-4: Individual and Pair wise Comparison of mean levels of RANK-L

\section{DISCUSSION}

The most significant finding of the present work was that psychological stress has a negative impact on orthodontic tooth movement by causing a reduction in its rate and osteoclastic activity and honey has no significant correlation with stress levels, hence does not improve orthodontic tooth movement efficiency. The rat model was chosen due to its easy handling, cost-effectiveness, and easy availability. Female rats were selected due to their increased sympathoadrenal and hypothalamicpituitary-adrenal (HPA) axis reactivity to stressful stimuli. ${ }^{14}$

The present study was conducted to demonstrate the effects of psychological stress with Stress reliever (Honey) on OTM in a rat model by measuring the magnitude of OTM, number of Osteoclasts and Levels of Osteoclastic marker i.e., RANKL. This Protocol of stress induction was following the study conducted by Mirzakouchaki et al., 2011.6

Evaluation of the results of Orthodontic Tooth Movement in this study showed that the mean orthodontic tooth movement in the control group was significantly higher as compared to PS and PSH groups. These results were supported by the study conducted by Ahmed Akhoundi et al., 2020, in which a strong linear escalation in tooth movement was noted in the orthodontically treated non-stressed group. ${ }^{15}$ Mirzakouchaki et al also showed that Psychological Stress reduces OTM. ${ }^{6}$

No significant difference in the mean osteoclastic count among groups ( $\mathrm{p}$-value $=0.128$ ) was found. The conflicting result was observed in the experimental study conducted by Mirzakouchaki et al., 2011. Under a light microscope reduced osteoclastic count in the surface area was observed on the 7th day of spring placement because of psychological stress as compared to the control group ( $\mathrm{p}$-value $=1$ ). However, on the 14th day of spring activity, in the experimental group tooth movement as compared to the control group was less but the difference in osteoclast count among the two was not statistically significant on day 14 . This showed that psychological stress lessens the number of osteoclasts but this decrease was not linear. Similar results were presented by Liu et al., 2020. ${ }^{16}$ The increase in the RANKL level signifies increased Osteoclastic activity. In the current study, there was a fair and statistically significant rise in control group levels as compared to the other experimental groups. This shows increased action of the osteoclasts permitting adequate bone remodeling during uninterrupted orthodontic tooth movement. RANKL is a fundamental factor in boosting osteoclastogenesis. ${ }^{17}$ Castro et al., 2020 found out in their experiment that, on the compression side of orthodontic force there is expression of RANKL in bone and periodontal ligament hence promoting the bone resorption activity. ${ }^{18}$

The strength of this experimental research on animal model is that control over variables and histological findings can be achieved.

The weaknesses of this experimental research is, it is an animal model and should be replicated on humans with a larger sample size and for longer durations.

\section{CONCLUSION}

Psychological stress decelerates orthodontic tooth movement by causing a reduction in its rate and osteoclastic activity and honey has no significant correlation with lowering stress levels, hence does not improve orthodontic tooth movement efficiency. The recommendations for clinicians are to rule out the stress conditions before starting the orthodontic cases.

\section{REFERENCES}

1. Xu L, Sun X, Zhu G, Mao J, Baban B, Qin X. Local delivery of simvastatin maintains tooth anchorage during mechanical tooth moving via antiinflammation property and AMPK/MAPK/NF-kB inhibition. J Cell Mol Med. 2021; 25(1):333-44.

2. Yamaguchi M, Garlet GP. Inflammatory Response in the Periodontal Ligament and Dental Pulp During Orthodontic Tooth Movement. In: Biological Mechanisms of Tooth Movement. John Wiley \& Sons, Ltd; 2021; 49-67. 
3. Krishnan V, Davidovitch Z, Kuijpers-Jagtman AM. Biological Orthodontics. In: Biological Mechanisms of Tooth Movement. John Wiley \& Sons, Ltd; 2021; 217-37.

4. Santana L, Duarte-Rodrigues L, Alves Duarte A, Galvão E, Douglas de Oliveira D, Marques LS, Falci SGM. Systematic review of biological therapy to accelerate orthodontic tooth movement in animals: Translational approach. Arch Oral Biol. 2020; 110: 104597.

5. Assad S, Khan HH, Ghazanfar H, Khan ZH, Mansoor S, Rahman MA, Khan GH, Zafar B, Tariq U, Malik SA. Role of Sex Hormone Levels and Psychological Stress in the Pathogenesis of Autoimmune Diseases. Cureus. 2021; 9(6).

6. Mirzakouchaki B, Firoozi F, Firoozi S. Effect of psychological stress on orthodontic tooth movement in rats. Med Oral Patol Oral Cirugia Bucal. 2011; e285-91.

7. Samarghandian S, Farkhondeh T, Samini F. Honey and Health: A Review of Recent Clinical Research. Pharmacogn Res. 2017; 9(2):121-7.

8. Benelli G, Pavela R, Petrelli R, Cappellacci L, Bartolucci F, Canale A, et al. Origanum syriacum subsp. syriacum: From an ingredient of Lebanese 'manoushe' to a source of effective and eco-friendly botanical insecticides. Ind Crops Prod.2019;134:26-32.

9. Arshad N 'Ain, Lin TS, Yahaya MF. Stingless Bee Honey Reduces Anxiety and Improves Memory of the Metabolic Disease-induced Rats. CNS Neurol Disord - Drug Targets- CNS Neurol Disord. 2020; 19(2):115-26.

10. Ilyas M, Fahim A, Awan U, Athar Y, Sharjeel N, Arshad A, et al. Effect of Honey on Healing of Extracted Tooth Socket of Albino Wista Rats. Int Med J 1994. 2015; 22:422-5.

11. $\mathrm{Pu} \mathrm{H}$, Hua $\mathrm{Y}$. Hydrogen sulfide regulates bone remodeling and promotes orthodontic tooth movement. Mol Med Rep. 2017 Dec; 16(6):9415-22.

12. Sami W, Alrukban M, Waqas T, Asad M, Afzal K. Sample Size Determination In Health Research. J Ayub Med Coll Abbottabad JAMC. 2018;30:308-11.

13. guide-for-the-care-and-use-of-laboratory-animals. [cited 2021 Jan 28]. Available from: https:/grants.nih.gov/grants/olaw/guide-for-the-careand-use-of-laboratory-animals.pdf

14. Rincón-Cortés M, Herman JP, Lupien S, Maguire J, Shansky RM. Stress: Influence of sex, reproductive status and gender. Neurobiol Stress.2019;10:100155.

15. Ahmad Akhoundi MS, Shaygan-Mehr M, Keshvad MA, Etemad Moghaddam S, Alaeddini M, Dehpour A, et al. Effect of amitriptyline on orthodontic tooth movement in rats: an experimental study. J Dent Res Dent Clin Dent Prospects. 2020; 14(3):147-52.

16. Liu X, Wang X, Zhang L, Yang F, Nie F, Zhang J. Inhibitory effects of resveratrol on orthodontic tooth movement and associated root resorption in rats. Arch Oral Biol. 2020; 111:104642.

17. Kelly RR, McDonald LT, Jensen NR, Sidles SJ, LaRue AC. Impacts of Psychological Stress on Osteoporosis: Clinical Implications and Treatment Interactions. Front Psychiatry. 2019; 10:200.

18. Castro MML, Ferreira $\mathrm{R}$ de $\mathrm{O}$, Fagundes NCF, Almeida APCPSC, Maia LC, Lima RR. Association between Psychological Stress and Periodontitis: A Systematic Review. Eur J Dent. 2020; 14(1):171-9.

\section{The Authors:}

Dr. Reem Abid

Postgraduate Trainee,

Department of Orthodontics,

de'Montmorency College of Dentistry, Lahore.

Dr. Muhammad Sharjeel Ilyas,

Associate Professor

Department of Oral Biology,

Post Graduate Medical Institute, Lahore.

Dr. Ghazala Rubi

Director Research Lab.,

Central Research Laboratory,

Postgraduate Medical Institute, Lahore.

Dr. Amna Ahsan

Assistant Professor

Department of Oral Biology,

Post Graduate Medical Institute, Lahore.

Dr. Sadia Shakeel

Assistant Professor

Department of Oral Biology,

Post Graduate Medical Institute, Lahore.

Dr. Arfa Ahmed

Postgraduate Trainee,

Department of Orthodontics,

de' Montmorency College of Dentistry, Lahore.

\section{Corresponding Author:}

Dr. Muhammad Sharjeel Ilyas,

Associate Professor

Department of Oral Biology,

Post Graduate Medical Institute, Lahore.

E-mail: sharjeel_ilyas@hotmail.com 